Ann. Sci. For., 1985, 42 (4), 435-452

\title{
Essai et comparaison de trois méthodes de classement de surface de bois massif pour leur rugosité : méthodes pneumatique et sensorielles
}

\author{
F. MOTHE \\ I.N.R.A., Station de Recherches sur la Qualité des Bois \\ Centre de Recherches forestières de Nancy, Champenoux, F 54280 Seichamps
}

\section{Résumé}

18 éprouvettes de bois massif représentant 3 essences croisées avec 5 catégories d'usinage ont été classées pour leur rugosité (face radiale - longitudinale) suivant trois modalités :

VISO : test visiotactile classique ;

TACT : test tactile pur se déroulant dans l'obscurité ;

PNEU : rugosimètre pneumatique fonctionnant par fuite d'air.

Les résultats, traités par analyse des correspondances, montrent sur le plan méthodologique que la répétabilité des mesures fournies par l'appareil pneumatique est excellente, mais que ce dernier est plus sensible aux propriétés du plan ligneux (présence de gros vaisseaux en particulier) qu'aux caractéristiques de l'usinage. D'autre part, l'existence d'un effet de la lumière sur les classements visiotactiles a été constatée : les échantillons de merisier apparaissent plus rugueux en absence de lumière alors que la rugosité du chêne rouge est surestimée lorsque les échantillons sont visibles.

Une des propriétés importantes du bois, notamment pour ses utilisations les plus nobles, est son état de surface qui conditionne son aspect esthétique, mais aussi l'importance des pertes de matière lors des phases de finition du matériau. La rugosité est l'un des critères qui permet de définir l'état d'une surface : aussi a-t-elle depuis longtemps été prise en compte par les chercheurs américains, et plus récemment au Japon et en Europe.

\section{Introduction}

Un grand nombre de méthodes de mesure ou d'estimation de la rugosité du bois ont été testées (voir à ce sujet MARchaL, 1983), et plusieurs auteurs se sont attachés à les comparer entre elles. 
Le but de ces travaux est la mise au point d'une technique de mesure à la fois fiable, rapide et peu coûteuse, dont le besoin se fait sentir tant en laboratoise que dans l'industrie. L'appareil pneumatique que nous expérimentons ici présente indéniablement ces deux dernières qualités : coût de fabrication peu ćlevé, rapidité des mesures (quelques secondes par mesure). Il est de plus facile à manipuler, peu fragile, et offre l'avantage d'effectuer des mesures tridimensionnelles (par opposition aux appareils travaillant sur un profil de surface, à deux dimensions). Il reste à répondre aux deux questions suivantes :

- lal "rugosité » que mesure l'appareil est-elle identique à la rugosité sensorielle telle que la perçoit la peau humaine? Est-elle corrélée à l'un des critères de rugosité classiques?

- dans le cas contraire, les mesures effectuées permettent-clles de prédire la qualité du matériau pour une application donnée?

La démarche la plus courante pour répondre à de telles questions consiste à comparer les résultats obtenus avec ceux donnés par les deux principales méthodes de référence : le palpage mécanique de la surface par un stylet et les techniques manuelles d'estimation de la rugosité (méthodes visiotactiles). C'est cette dernière méthode que nous utilisons ici.

En outre, il nous a semblé intéressant de profiter de cette occasion pour étudicr ces méthodes visiotactiles et pour essayer d'expliquer quels facteurs sont pris en compte lorsque l'on classe manuellement des échantillons de bois pour leur rugosité.

Peu d'auteurs se sont intéressés à cette question. Les limites de la perception ont cependant été estimées par Schmaltz (1936) à une rugosité de $0,5 \mu$, voire même 0,1 lı avec de l'entraînement, pour le toucher, et à 1 lı pour la vue. Deux études récentes ont permis d'améliorer nos connaissances dans ce domaine :

SADoH et al. (1977) ont fait tester 82 échantillons de bois d'essences diverses (les trois quarts étant des leuillus) et ont observé des corrélations différentes pour les feuillus et pour les résineux entre la rugosité sensorielle et les propriétés anatomiques du bois. La sensation de rugosité serait due, pour les feuillus, aux vaisseaux, alors que ce sont les ondulations provoquées par les cernes qui seraient prises en compte pour les résineux.

D'autres auteurs japonais (YASUDA et al., 1983) ont fait qualifier par une cinquantaine de personnes l'état de surface d'éprouvettes de bois massif représentant 11 essences feuillues. Les testeurs devaient choisir l'un ou l'autre de chaque couple de qualificatifs antagonistes qui leur étaient proposés (doux/rugueux, fin/grossier, clair/sombre, ...). Les résultats, traités par analyse des composantes principales, ont permis de définir trois facteurs entrant en ligne de compte pour l'estimation d'une surface : le premier avait trait à la rugosité, le second à la régularité, et le troisième à la clarté du dessin. Les auteurs ont obtenu une bonne corrélation entre le premier facteur et la rugosité géométrique (RMS) mesurée par un appareil à palpeur.

Nous essayerons ici de compléter ces connaissances en nous atlachant surtout à déterminer le rôle joué par la lumière dans un test visiotactile. 


\section{Matériel expérimental}

L'expérience a porté sur 18 éprouvettes de bois massif (dimensions : $45 \times 200$ $\times 20 \mathrm{~mm}^{3}$ ). Trois essences étaient représentées par 6 éprouvettes chacune, usinées selon 5 modalités (tabl. 1).

TABLEAU 1

Description des échantillons.

Description of samples.

\begin{tabular}{|c|c|c|c|c|}
\hline Essence & Désignation & Usinage (1) & $\begin{array}{l}\text { Nbre de cernes } \\
\text { sur la face usinée }\end{array}$ & $\begin{array}{l}\text { Densité (2) } \\
\left(\mathrm{g} / \mathrm{dm}^{3}\right)\end{array}$ \\
\hline Prunus avium & $\begin{array}{l}\text { K } \\
F \\
\text { L } \\
O \\
G \\
\text { E }\end{array}$ & $\begin{array}{c}\text { P } 80 \\
\text { Brut } \\
\text { Brut } \\
\text { P } 120 \mathrm{~N} \\
\text { P } 120 \mathrm{~V} \\
\text { P } 150+ \\
\text { polissage manuel } \\
\end{array}$ & $\begin{array}{l}16 \\
13 \\
14 \\
24 \\
17 \\
14\end{array}$ & $\begin{array}{l}540 \\
557 \\
537 \\
540 \\
564 \\
570\end{array}$ \\
\hline Quercus robur & $\begin{array}{l}P \\
N \\
H \\
J \\
B \\
R\end{array}$ & $\begin{array}{c}\text { P } 80 \\
\text { Brut } \\
\text { Brut } \\
\text { P } 120 \mathrm{~N} \\
\text { P } 120 \mathrm{~V} \\
\text { P } 150+ \\
\text { polissage manuel } \\
\end{array}$ & $\begin{array}{l}12 \\
16 \\
13 \\
17 \\
15 \\
17\end{array}$ & $\begin{array}{l}773 \\
739 \\
756 \\
755 \\
769 \\
743\end{array}$ \\
\hline Quercus rubra & $\begin{array}{c}\mathrm{D} \\
\mathrm{Q} \\
\mathrm{I} \\
\mathrm{A} \\
\mathrm{M} \\
\mathrm{C}\end{array}$ & $\begin{array}{c}\text { P } 80 \\
\text { Brut } \\
\text { Brut } \\
\text { P } 120 \mathrm{~N} \\
\text { P } 120 \mathrm{~V} \\
\text { P } 150+ \\
\text { polissage manuel }\end{array}$ & $\begin{array}{l}20 \\
19 \\
18 \\
17 \\
15 \\
12\end{array}$ & $\begin{array}{l}591 \\
630 \\
742 \\
634 \\
735 \\
773\end{array}$ \\
\hline \multicolumn{5}{|c|}{$\begin{array}{l}\text { Usinage - Surface preparation. } \\
\text { Nombre de cernes sur la face usinée - Ring number on the milled surface. } \\
\text { Brut - Rough. } \\
\text { Polissage manuel - Manual sanding. } \\
\text { Brut de rabotage - After planing without sanding. } \\
\text { Grain de ponçage } 80 \text { à } 150-80 \text { to } 150 \text { grain of sanding. } \\
\text { Bande de ponçage neuve - New sanding belt. } \\
\text { Bande de ponçage usée : Not new sanding bell. }\end{array}$} \\
\hline
\end{tabular}


Le ponçage a été effectué à la ponceuse à bande, sur une seule face de chaque échantillon, dans le plan radial-longitudinal $\left(45 \times 200 \mathrm{~mm}^{2}\right)$. Ce matériel se veut représentatif des états de surface que risque de rencontrer dans notre Station de Recherches le rugosimètre pneumatique. Le choix des essences est en partie motivé par l'importance accordée à leur état de surface par certains utilisateurs. Le nombre d'espèces et l'échantillonnage de celles-ci sont cependant insuffisantes pour permettre l'estimation des variabilités inter et intraspécifique; aussi, nous abstiendrons-nous par la suite de tirer des conclusions sur la qualité générale des essences utilisées du point de vue de la rugosité.

L'origine des éprouvettes est d'ailleurs mal connue; on sait cependant que les chênes pédonculés proviennent de la forêt de Citeaux (Côte-d'Or) et que les éprouvettes $H, R$ et $P$ sont issues du même arbre. Les échantillons $D, A, Q$ de chêne rouge ont été découpés dans la même planche d'origine malheureusement inconnue. Les autres chênes rouges proviennent de la forêt de Chaux (Jura).

A l'exception de l'éprouvette L, les merisiers proviennent tous de deux arbres isolés de Champenoux (Meurthe-et-Moselle).

\section{Expérimentation}

Le classement pour la rugosité des 18 éprouvettes, toutes essences confondues, a été effectué suivant trois modalités (tabl. 2).

VISO : 18 personnes ont tout d'abord été sollicitées pour classer manuellement les éprouvettes à leur convenance, avec pour seule restriction de ne jamais en placer deux au même rang. Les éprouvettes étaient toujours présentées dans le même ordre, dans une pièce éclairée à la lumière naturelle.

TACT : les 18 mêmes personnes ont accepté de répéter le test dans des conditions différentes :

- obscurité presque complète,

-- ordre des éprouvettes au départ modifié.

PNEU : la rugosité a enfin été mesurée par l'appareil décrit dans les articles de T. Bonac $(1975,1979)$, appareil dont une copic a été réalisée à la Station par J.R. Perrin, J. Perrin et F. Thiercelin.

L'air sous pression est insufflé par une buse pesant $500 \mathrm{~g}$ et terminée par 19 tubes (diamètre intérieur $3 \mathrm{~mm}$ ) que l'on pose sur la surface (fig. 1). La rugosité "absolue " est déterminée par la chute de pression enregistrée sur colonne d'eau au niveau de cette buse; cette chute de pression sera d'autant plus forte que la surface est rugueuse.

La pression d'entrée - environ $50 \mathrm{~mm} \mathrm{CE} \mathrm{-} \mathrm{(CE} \mathrm{=} \mathrm{colonne} \mathrm{d'eau})$ n'est pas parfaitement régulée. Un réglage périodique est donc nécessaire lorsque l'appareil est utilisé de façon prolongée ou lorsqu'un long intervalle s'écoule entre deux séries de mesures. Dans l'état actuel, ce réglage s'effectue en plaçant l'appareil au contact d'une surface de verre polie; il suffit ensuite de manœuvrer une vanne placée en avant de la buse pour modifier la pression. Ce réglage est malheureusement im- 


\section{TABLEAU 2}

Description des trois modalités de classement:

VISO : visiotactile; TACT : tactile pur; PNEU : pneamatic.

Description of the three methods of grading :

VISO : visual and tactile; TACT : tactile: PNEU : pneumatic.

\begin{tabular}{|c|c|c|c|}
\hline Nature du test & Test visiotactile & Test tactile pur & $\begin{array}{l}\text { Rugosimètre } \\
\text { pneumatique }\end{array}$ \\
\hline ldentification & VISO & ТАCГ & PNEU \\
\hline Nombre de tests .......... & 23 & 18 & 18 \\
\hline Nombre d'opérateurs ....... & 18 & 1 à 9 te & opérateur \\
\hline $\begin{array}{l}\text { Ordre des échantillons au début } \\
\text { du test } \ldots \ldots \ldots \ldots \ldots \ldots \ldots\end{array}$ & Constant & Constant & Variable \\
\hline$\ldots \ldots \ldots \ldots \ldots$ & Non a & orisés & Fréquents \\
\hline Remarques & Lumière naturelle & Chambre noire & $\begin{array}{l}\text { - } 2 \text { mesures par } \\
\text { échantillon } \\
\text { - Problème } \\
\text { de référence }\end{array}$ \\
\hline \multicolumn{4}{|c|}{$\begin{array}{l}\text { Nature du test - Type of test. } \\
\text { Test tactile pur - Pure risiolactile test. } \\
\text { Rugosimètre pneumatique - Pnematic ronghnessmeter. } \\
\text { Nombre de tests - Test number. } \\
\text { Nombre d'opérateurs - Tester number. } \\
\text { Ordre des échantillons au début du test - Rank of samples before } \\
\text { Lumière naturelle - Natural light. } \\
\text { Chambre noire - Room in darkizess. } \\
2 \text { mesures par échantillon - } 2 \text { measures in each sample. } \\
\text { Problème de référence - Problem of rejerence. }\end{array}$} \\
\hline
\end{tabular}

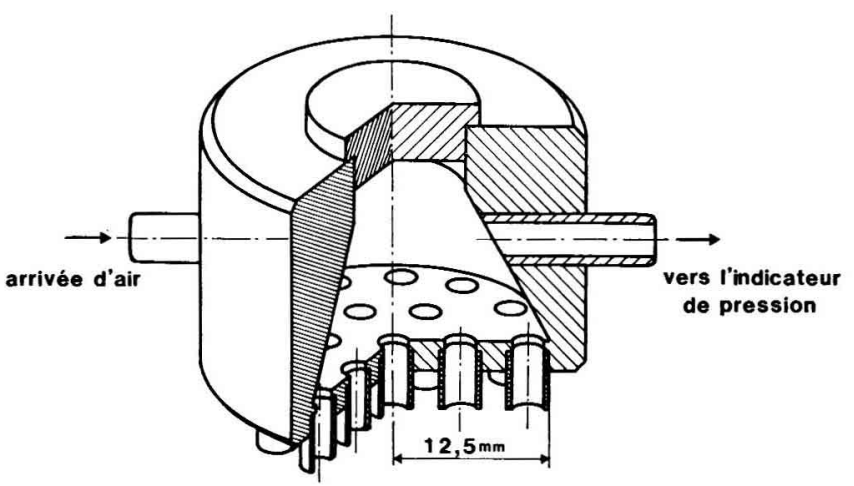

FIG. 1

Buse du rugosimètre pneumatique (d'après BonAC, 1979).

Pneumatic roughnessmeter nozzle (from BonAC, 1979).

Arrivée d'Air - Air supply.

Vers l'indicateur de pression - To the pressure gauge. 
précis - aussi n'avons-nous considéré que le classement des éprouvettes sans teni: compte de la valeur "absolue » de la rugosité (chute de pression moyenne exprimée en $\mathrm{cm}$ de colonne d'cau).

Nous avons adopté la démarche suivante : plusieurs séries de mesures identiques ont été effectuées, portant chacune sur les 18 éprouvettes, à raison de deux mesures par éprouvette. Il est peu probable que la pression d'entrée ait beaucoup varié au cours de ces 36 mesures, et donc que le classement des surfaces en fonction de la chute de pression enregistrée ait été influencée par ce facteur. Entre deux séries de mesures, l'appareil était volontairement déréglé, puis réétalonné au moyen de la surface de référence.

Dix-huit séries de mesures ont été effectuées de cette manière, conduisant ainsi à 18 classements des éprouvettes. Il faut noter que quatre manipulateurs ont été mis à contribution qui ont effectué un nombre variable de séries de mesures. $\mathrm{Ce}$ nombre varie de un à neuf.

\section{Comparaison des trois méthodes de classement par l'analyse factorielle des correspondances}

L'analyse factorielle des correspondances (AFC) est une méthode de calcul proche de l'analyse des composantes principales. Ces deux types d'analyses ont une signification et des résultats généralement équivalents. Ils diffèrent surtout par les règles d'interprétation et la présentation des résultats (J. BAchacou) qui nous ont paru mieux adaptées à notre problème.

Les trois modalités présentées ci-dessus ont conduit à un certain nombrè de classements des mêmes éprouvettes; l'AFC prendra en compte tous ces classements de façon indépendante, ce qui permettra de vérifier la cohésion des tests appartenant à la même modalité. Mais, afin d'établir auparavant un classement-lype qui corresponde à chacune des trois méthodes : VISO, TACT et PNEU, nous avons effectué le calcul préliminaire suivant :

Une note correspondant à la moyenne des rangs obtenus dans la modalité a été attribuée à chacune des éprouvettes. Ces notes ont été ordonnées de façon à ce que l'éprouvette ayant obtenu la note moyenne la plus faible (forte rugosité) se voit attribuer le rang 1, l'éprouvette ayant reçu la note la plus élevée obtenant le rang 18.

Les trois classements résultant (tabl. 3) nous montrent que la répétition du test visiotactile dans l'obscurité n'a pas apporté de modifications importantes, mais que les classements sensoriels et pneumatique sont indéniablement différents.

Une telle différence n'est pas un obstacle en soi à l'exploitation de l'appareil pneumatique; l'objectif de cette étude méthodologique est justement de préciser son domaine d'application. Il aurait été utile de vérifier dans un premier temps si la ressemblance entre les classements sensoriels et pneumatique pouvait être améliorée sur une gamme de lugosité restreinte (par exemple, pour une essence donnée). Malheureusement, le matériel dont nous disposons est insuffisant pour répondre à cette question. Même s'il s'avérait qu'un échantillonnage différent conduise aux mêmes résultats, il est possible que les caractéristiques de surface responsables de la chute de pression enregistrée par l'appareil définissent parallèlement la qualité du 
matériau pour certaines applications. Le rugosimètre serait alors parfaitement adapté pour déterminer l'aptitude d'une éprouvette à l'emploi considéré. La comparaison des classements sensoriels et pneumatique devrait nous renseigner sur ces facteurs intervenant dans la mesure pneumatique, et par là même, nous aider à déterminer les applications du bois dans lesquelles ces propriétés interviennent.

\section{TABLEAU 3}

Classements par ordre de rugosité décroissante résultant des trois modalités (VISO, TACT, PNEU).

Classification in decreasing order of roughness for the three methods (VISO, TACT. PNEU).

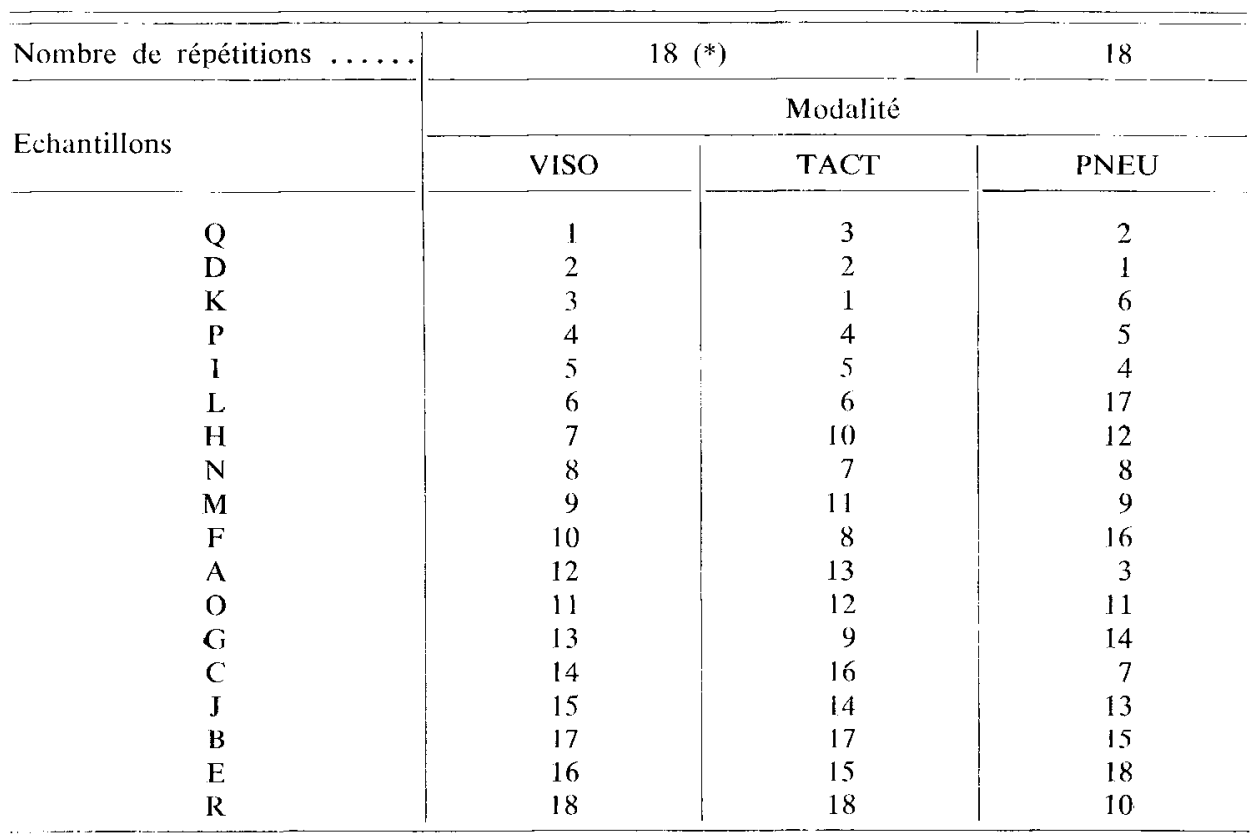

(*) Les 18 mêmes personnes ont participé aux deux modalités. The 18 same testers participated in two testing modalities.

Auparavant, il importe de vérifier que les divers classements fournis par le rugosimètre sont suffisamment cohérents entre eux pour attester de la fiabilité des mesures pneumatiques.

\subsection{Répétabilité des classements obtenus par la même méthode}

Pour effectuer cette première analyse factorielle des correspondances, les données sont présentées sous la forme d'un tableau croisé dont les colonnes figurent les 18 éprouvettes et les lignes les 18 rangs attribuables. A l'intersection d'une ligne $R$ et d'une colonne $E$ est noté le nombre de testeurs ayant attribué le rang $R$ à l'éprouvette $\mathrm{E}$. 
Deux ensembles de variables liées entre elles ont ainsi été créées : les variables rangs et les variables éprouvettes. L'intensité de la liaison entre ces variables dépend évidemment de l'expérience. Ainsi :

a) deux éprouvettes seront proches l'une de l'autre si elles ont été classées sensiblement aux mêmes rangs avee la même fréquence;

b) deux rangs seront proches l'un de l'autre s'ils ont été attribués avec une fréquence équivalente aux mêmes éprouvettes;

c) une éprouvelte est proche d'un rang qui lui a été attribué avec une fréquence supéricure à la moyenne.

Les graphiques résultant de l'AFC permettent de visualiser physiquenent ces variables; chacune d'elles est représentée par un point et la proximité entre deux points traduit l'intensité de la liaison entre les variables qu'ils symbolisent.

Bien entendu, une telle représentation ne peut pas forcément se restreindre à un espace à deux dimensions. Nous n'exploiterons cependant ici que la projection du nuage des points sur le plan des deux axes principaux définis par le calcul, ceux qui permettent la meilleure ségrégation des points variables.

L'AFC permet d'adjoindre au tableau des données des variables supplémentaires qui n'interviennent pas dans la détermination des axes d'inertie mais dont les coordonnées sont néanmoins calculées dans le plan déjà défini. Les huit variables

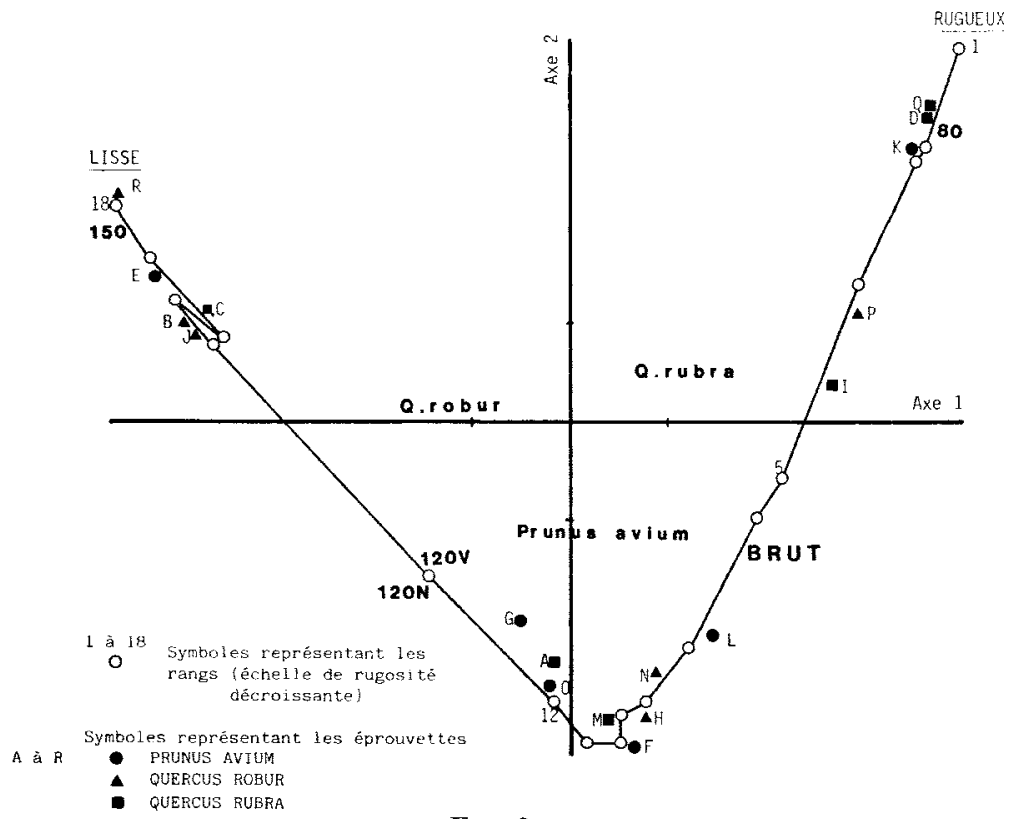

FIG. 2 a

Première analyse des correspondances entre les échantillons (colonnes) et les rangs obtenus dans les modalités VISO et TACT (lignes; 36 répétitions). Représentation simultanée des lignes et des colonnes

dans le repère formé par les deux premiers axes d'inertie.

First correspondance analysis between samples (columns) and ranks obtained for VISO and TACT (lines; 36 repetitions).

Joint representation of lines and columns in the plane of the first two axes of inertia. 
supplémentaires que nous avons introduites représentent des sous-groupes d'éprouvettes (merisier - chênes rouges - chênes pédonculés - et les cinq catégories d'usinage) qui seront ainsi symbolisés chacun par un point sur le graphe résultani. Les coordonnées de ce point sont celles du barycentre des points symbolisant les éléments du sous-groupe alfectés chacun d'une inertie dépendant des axes de représentation.

L'analyse a été effectuée une première fois (fig. 2 a) en ne tenant compte que des modalités VISO et TACT dont les résultats sont peu différents. La modalité PNEU a fait l'objet d'une analyse séparée (fig. 2 b).

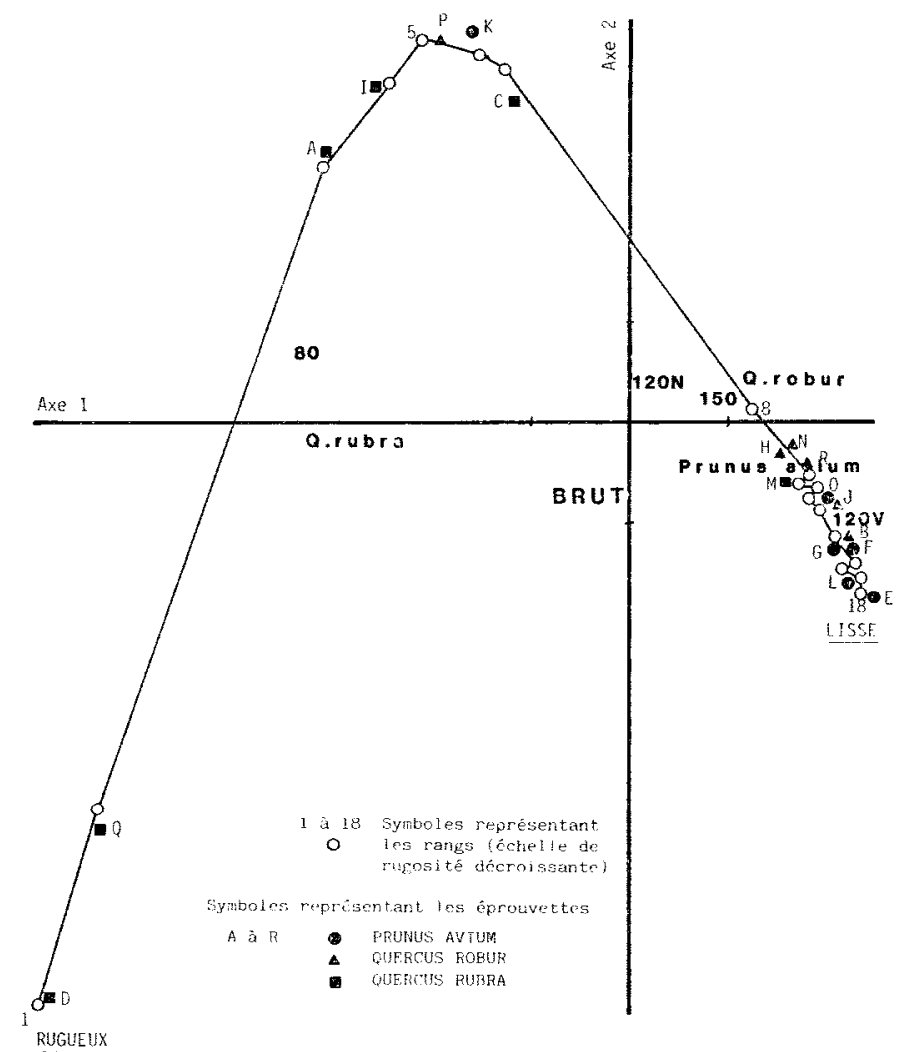

Fig. $2 b$

Premiere analyse des correspondances entre les échantillons (colomes) et les rangs obtenus dans la modalité PNEU (lignes; 18 répétitions). Représentation simultanée des lignes et des colonnes: dans le repère formé par les deux premiers axes d'inertie.

First correspondance analysis between samples (columns) and ranks obtained for PNEU (lines; 18 repetitions).

Joint representation of lines and columns in the plane of the first wo axes of inertia.

Lisse - Smooth.

Rugueux : Rough.

Symboles représentant les rangs (échelle de rugosité décroissantc) - Symbols for ranks (decreasing roughness scale).

Symboles représentant les éprouvettes - Symbols for samples. 
On constate sur ces ligures que la courbe reliant les rangs entre cux rappelle la forme d'une parabole (l'orientation de celle-ci ne présente pas de signification particulière). Les individus placés à l'intérieur de cette courbe sont ceux dont le classement a été particulièrement variable si l'on se réfère à la règle c) énoncée précédemment. Cette remarque s'applique également aux variables supplémentaires qui symbolisent les classes d'essences et d'usinages.

La dissymétrie de la figure 2 b est significative : à chacun des rangs 1 à 7 (forte rugosité) correspond une éprouvette bien définie (étiquetées D, Q, A, I, P, K et C.), ce qui signifie que le classement de ces sept surfaces a peu varié d'un test pneumatique à l'autre. En revanche, les rangs 8 à 18 et les 10 éprouvettes restantes forment un nuage de points compact dans lequel l'appariement éprouvette-rang n'apparaît pas clairement.

La figure 2 a est quant à elle presque symétrique; les éprouvettes comme les rangs se répartissent équitablement au long de la courbe. On note qu'un ensemble de 5 éprouvettes (R, E, B, I et C) est groupé autour du pôle de faible rugosité, alors que $Q, D, K, P$ et I forment une classe rugueuse.

Un premier caractère distinctif apparaît ainsi clairement entre les méthodes manuelles et pneumatique : le rugosimètre classe de façon moins variable les éprouvettes les plus rugueuses (du point de vue pneumatique), mais son pouvoir de ségrégation semble décroître en deçà d'un certain seuil de rugosité qui n'affecte pas le sens du toucher.

L'origine d'un tel seuil serait probablement à chercher dans le principe de fonctionnement de l'appareil pneumatique. Lorsque la porosité est faible, l'épaisseur des parois des tubes d'injection peut en effet devenir suffisante pour occulter toutes les ouvertures susceptibles de laisser passage à un flux d'air. La chute de pression enregistrée serait alors imputable aux seuls défauts de planéité qui, théoriquement, sont peu variables d'une éprouvette à l'autre.

\subsection{Facteurs essence et usinage}

La localisation des variables supplémentaires (classes d'essences et d'usinages) sur les figures $2 \mathrm{a}$ et $2 \mathrm{~b}$ apporte de nouvelles précisions. A l'exception des classes « $120 \mathrm{~V}$ » et « $120 \mathrm{~N}$ » qui sont confondues, la distribution uniforme des catégories d'usinages le long de la parabole de la figure 2 a nous montre que la qualité de la finition est facilement décelable au toucher. Les classes d'essences représentées au centre du graphique sont formées d'éléments jugés de façon très variable : le facteur essence a donc pu influencer les testeurs manucls.

Sur la figure $2 \mathrm{~b}$, la localisation des variables supplémentaires montre que le nuage de points situé à la droite du graphique rassemble des essences et des catégories d'usinage variées. Du côté opposé, on trouve les éprouvettes $\mathrm{K}, \mathrm{P}$ et $\mathrm{O}$ de la classe « $80 »$ (ponçage le plus grossier) et la quasi-totalité des chênes rouges. On peut conclure de cette répartition que le plan ligneux intervient de façon non négligeable dans le classement pneumatique bien que deux essences aussi différentes que le merisier et le chêne pédonculé obtiennent des rangs moyens équivalents. 


\subsection{Rugosité « sensorielle» et rugosité «pneumatique»}

L'importance variable accordée aux effets essence et usinage par l'homme et par la machine est probablement à l'origine des différences de classements observées dans le tableau 3. Cependant, pour mieux comprendre le fonctionnement de l'appareil pneumatique en liaison avec les caractéristiques de la surface, une comparaison plus poussée des deux méthodes de classement serait enrichissante.

Pour cela, nous utiliserons une nouvelle fois l'AFC en présentant les données sous une forme nouvelle; en premier lieu, puisqu'il s'agit d'une étude comparative, les classements effectués dans les trois modalités devront intervenir dans la même analyse. Nous souhaitons d'autre part comparer les classements obtenus et étudier la variation du rang d'une éprouvette d'un classement à l'autre. Les variables à considérer seront donc les suivantes :

- $3 \times 18$ variables-tests correspondant aux 54 classements effectués (VISO + TACT + PNEU) ;

- 18 variables-éprouvettes.

Ces deux ensembles de variables sont croisés; la valeur d'unc variable-test $T$ définie pour chaque éprouvette $\mathrm{E}$ correspond au rang obtenu par $\mathbf{E}$ dans le test considéré, et cette valeur est aussi celle d'une variable-éprouvette E définie par le test $\mathrm{T}$.

En pratique, une telle présentation n'est pas satisfaisante. En effet, unc variable E et une variable $\mathrm{T}$ sont liées si $\mathrm{E}$ obtient dans le test $\mathrm{T}$ un rang inhabituel («plus lisse» ou «plus rugueux» que la moyenne). Or, sur le graphique résultant d'une telle présentation des données, la proximité de deux points $E$ et $T$ signifierait seulement que $\mathrm{E}$ a été classé «peu rugueux» par $\mathrm{T}$. Le cas inverse (E particulièrement rugueux) se traduirait au contraire par l'éloignement des points $\mathrm{E}$ et $\mathrm{T}$.

Pour obtenir une représentation symétrique des résultats, chacune des variableséprouvettes $E$ prendra deux valeurs pour chaque test $T$ :

son rang $\mathrm{E}^{+}$- obtenu sur une échelle de rugosité décroissante,

son rang $\mathrm{E}^{-}$obtenu sur une échelle de rugosité croissante.

Nous pouvons alors énoncer quelques règles de lecture de la ligure 3 (représentation dans le repère formé par les deux premiers axes d'inertic) :

a) deux points représentant des tests sont d'autant plus proches l'un de l'autre que les classements qui en sont issus sont ressemblants;

b 1) autour du pôle de forte rugosité d'une éprouvette $E$ (pôle négatil $\mathrm{E}^{-}$) sont rassemblés les tests ayant classé $\mathrm{E}$ plus rugueux que la moyenne ;

b 2) à l'inverse, les tests dans lesquels $E$ apparaît moins rugueux que la moyenne des autres tests, sont proches du pôle positif $\mathrm{E}^{+}$.

L'examen de la figure 3 montre que l'axe horizontal (axe 2) sépare les symboles représentant les tests en deux groupes : les tests sensoriels occupent la partic supérieure du graphe, alors que les tests pneumatiques sont rassemblés en un nuage plus compact au-dessus de l'axe 2. Nous pouvons en déduire que le rugosimètre pneumatique a offert lors de ces tests une répétabilité du classement globalement supérieure à celle des tests manuels, mais que les deux techniques ont abouti à des 
classements sensiblement différents. On vérifie par ailleurs l'absence d'effet manipulateur sur les classements pneumatiques (les quatre manipulateurs mis à contribution sont identifiés par les lettres «a » à « d » sur la figure 3 ).

Les règles b 1 et b 2 énoncées ci-dessus permettent d'apporter de nouvclles précisions. En effet, la répartition des pôles représentant les échantillons de part et d'autre de l'axe horizontal montrent que, d'une façon générale, les merisier's ont été jugés moins rugueux par l'appareil pneumatique que par les testeurs manuels

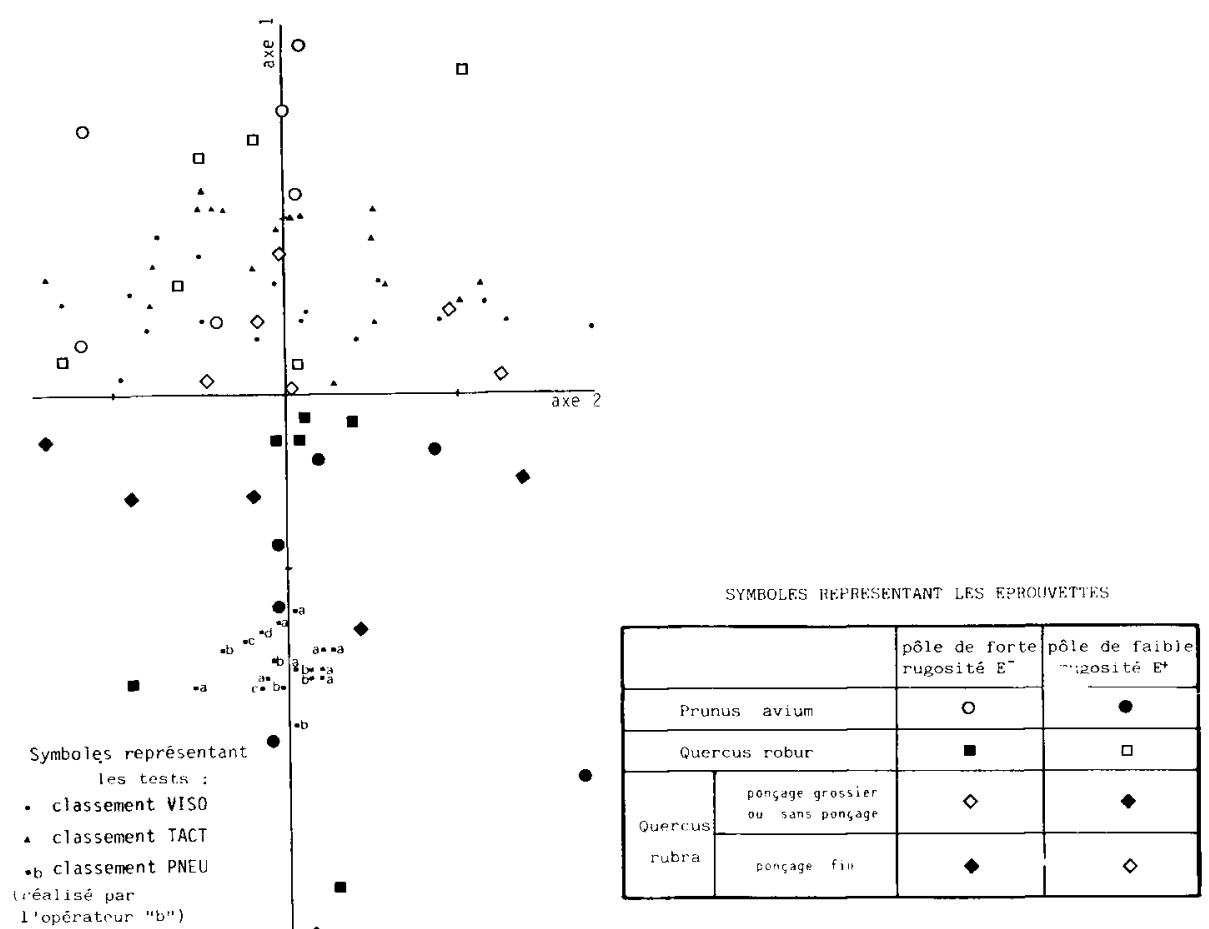

FIG. 3

Seconde analyse des correspondances entre les échantillons (colonnes)

et les 54 classements obtenus dans les trois modalités (VISO, TACT ¿t PNEU).

Représentation simultanée des lignes et des colonnes

dans le repère formé par les deax premiers axes d'inertie.

Second correspondance analysis between samples (columns)

and the 54 ranks obtained for three modalities (VISO, TACT and PNEU).

Joint representation of lines and columns in the plane of the first two axes of inertia.

Symboles représentant les tests - Symbols for ranks.

Classement - Rank.

Réalisé par l'opérateur b : Made by b tester.

Symboles représentant les éprouvettes - Symbols for samples.

Ponçage grossier ou sans ponçage - Rough sanding or without sanding.

Ponçage fin - Smooth sanding.

Pôle de forte rugosité $\mathrm{E}^{-}$- High roughness pole $E^{-}$.

Pôle de faible rugosité $\mathrm{E}^{-1}$ - Small roughness pole $E^{+}$. 
(tous les pôles de forte rugosité sont situés dans la partie supérieure de la figure 2). Le phénomène opposé s'observe avec les éprouvettes de chêne rouge qui sont classées "plus rugueuses" dans le test pneumatique. En ce qui concerne le chêne pédonculé, la qualité de l'usinage semble jouer un rôle puisque les trois surfaces les moins bien finies (brutes de rabotage ou poncées grossièrement) ont été jugées " plus rugueusess" au toucher.

Ce schéma peut se résumer de la façon suivante :

\begin{tabular}{|c|c|c|c|}
\hline & Merisier & Chêne pédonculé & Chêne rouge \\
\hline Ponçage fin & Classés \& moins & Classés « m & \multirow{2}{*}{$\begin{array}{l}\text { rugueux } \gg \text { par } \\
\text { VISO et TACT }\end{array}$} \\
\hline Ponçage grossier ... & rugueux $»$ & par PNEU & \\
\hline
\end{tabular}

Nous pouvons interpréter ceci de la façon suivante : pour l'appareil pneumattique, le classement des essences ne pose aucun problème; les gros vaisseaux du chêne rouge forment sur le plan radial - longitudinal des rainures longues de plusieurs centimètres qui provoquent des fuites d'air autrement plus importantes que la rugosité des plages de fibres. A l'opposé, le merisier, dont les pores sont diffus, est le plus lisse du point de vue pneumatique. La qualité de la finition, comme nous l'avons souligné, n'intervient ensuite que comme facteur de classification secondaite.

D'autre part, la taille des vaisseaux est insuffisante pour que la peau humaine les perçoive nettement. Le palpage manuel paraît beaucoup plus sensible aux microondulations - en particulier aux microcrêtes - qu'a provoquées le ponçage. On observe en conséquence un phénomène de nivellement des essences (merisier plus rugueux, chêne rouge plus lisse), et parallèlement une nouvelle ségrégation des éprouvettes apparaît qui concerne la qualité de la finition (bien poncé-plus lisse; peu poncé - plus rugueux).

\subsection{Rôle de la lumière dans un test visiotactile}

Un jugement visiotactile fait intervenir deux facultés sensorielles - la vue et le toucher - dont les effets, aux dires des personnes que nous avons sollicitées, sont parfois contradictoircs. On peut se demander, dès lors, s'il est rigourcux de comparer les résultats de test réalisés dans des conditions de luminosité différentes. C'est ce que nous avons voulu vérifier en répétant le test visiotactile (VISO) dans l'obscurité (TACT). Nous avons constaté précédemment que les classements résultant de ces deux modalités étaient en définitive peu différents (les nuages de points représentant les tests VISO et TACT sont confondus sur la figure 3). Nous navons cependant pas tenu compte jusqu'ici du fait que les tests TACT et VISO ont été effectués par les mêmes personnes.

L'AFC permet de traiter graphiquement cette question; il suffit pour cela de répéter l'analyse précédente en éliminant la modalité PNEU qui n'intervient pas ici. Afin de faciliter la lecture des graphiques, nous avons représenté séparément les nuages de points formés par les éprouvettes (fig. 4 a) et les tests (fig. 4 b). 


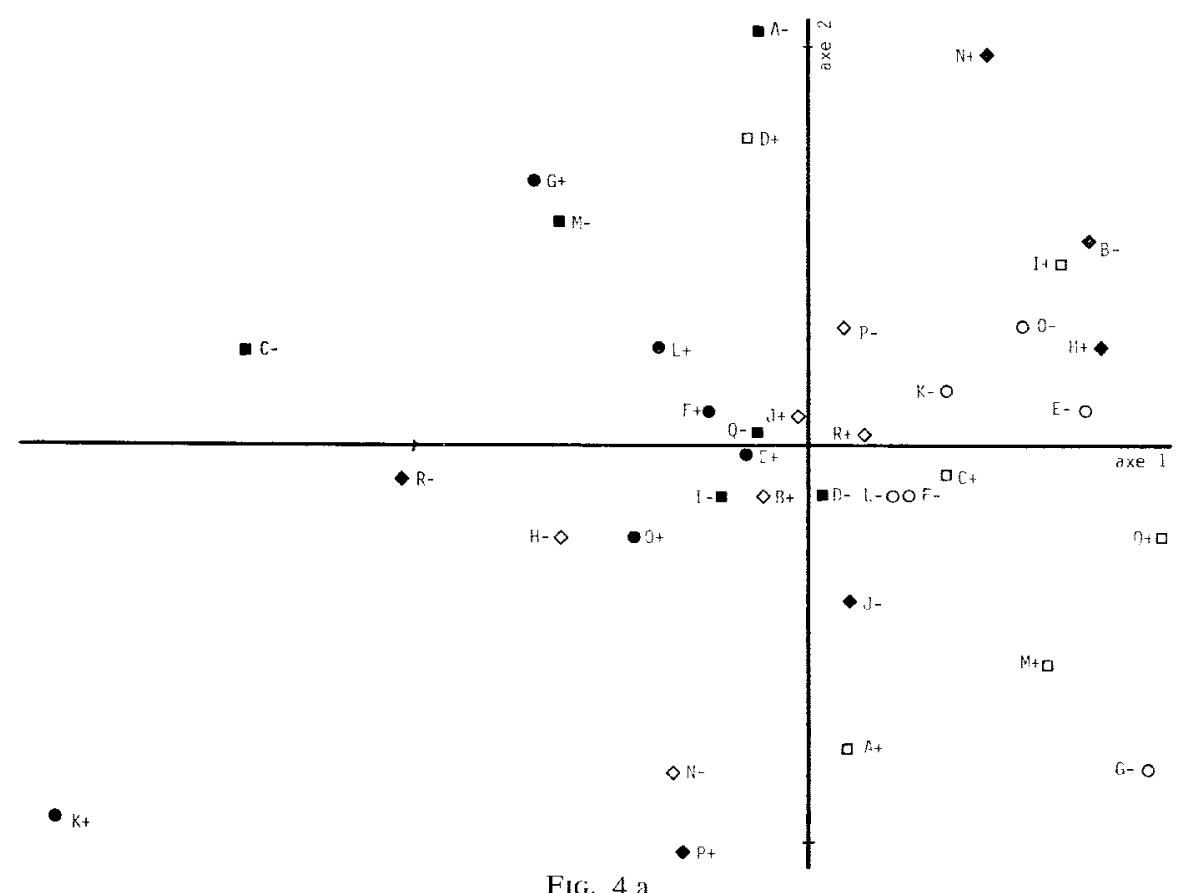

Fig. 4 a

Troisième analyse des correspondances entre les échantillons (colonnes) et les 36 classements VISO et TACT (lignes).

Représentation du nuage des colonnes dans le repère formé par les deax premiers axes d'inertie.

Third correspondance analysis between samples (columns) and the 36 VISO and TACT ranks (lines).

Representation of the columns cloud in the plane of the first wo axes of inertia.

Sur la figure $4 \mathrm{~b}$, les points représentant les deux tests effectués par la même personne sont reliés par une flèche orientée dans le sens $\mathrm{VISO} \rightarrow \mathrm{TACl}$. On visualise ainsi l'évolution du classement lorsque le test est répété dans l'obscurité.

Parmi les 18 flèches obtenues, 16 ont une composante horizontale positive et 14 une composante verticale négative. La résultante de ces flèches serait orientée dans le sens Nord-Ouest/Sud-Est. Il importe done d'étudier la répartition des pôles correspondant aux échantillons entre les deux zones que nous venons de définir.

Dans la région Sud-Est correspondant aux tests TACT, on constate une prédominance des pôles positifs du merisier (fig. 4 a), ce qui signifie que l'aspect visuel de la surface a contribué à améliorer le classement des éprouvettes de merisier (jugées «plus lisses »). Au contraire, les chênes rouges ont été classés «plus rugueux » lorsqu'on pouvait les voir en même temps que les toucher, comme le montre la prédominance des pôles négatifs de ces éprouvettes dans la région Sud-Est.

Etant donné qu'il s'agit ici de classements subjectifs. on est tenté d'expliquer ce phénomène par un effet psychologique : le testeur pardonnerait un peu au merisicr 
SYMBOLES REFFESENTANT LES EPROUVETTES Sur les FIGURES $4 \mathrm{a}$ et $4 \mathrm{~b}$

\begin{tabular}{|c|c|c|}
\hline & $\begin{array}{l}\text { Dôle de forte } \\
\text { rugosite } \mathrm{E}^{-}\end{array}$ & $\begin{array}{l}\text { pôle de faible } \\
\text { rugusite } \mathrm{r}^{+}\end{array}$ \\
\hline Prunus avium & 0 & - \\
\hline Quercus rubra & - & 口 \\
\hline 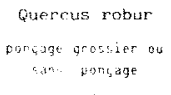 & $\diamond$ & $\bullet$ \\
\hline 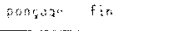 & $\diamond$ & $\diamond$ \\
\hline
\end{tabular}

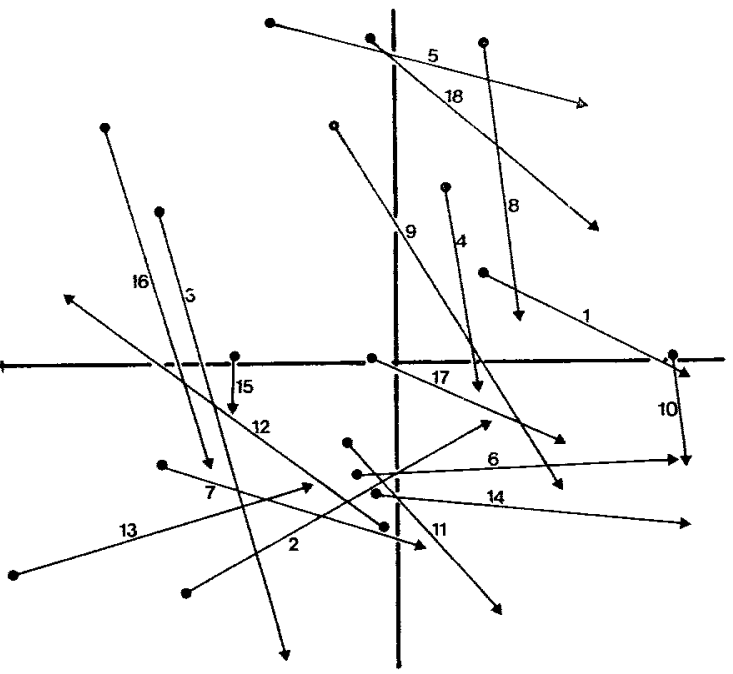

1 classement individuel viso.

classement TACT effectué par la même personne.

FIG. 4 b

Troisième analyse des correspondances entre les échantillons (colonnes) et les 36 classements VISO et TACT (lignes).

Représentation du nuage des lignes dans le repère formé par les deux premiers axes d'inertie.

Third correspondance analysis between samples (columns) and the 36 VISO and TACT ranks (lines).

Representation of the ranks cloud in the plane of the first two axes of inertia.

Classement individuel VISO - Individual VISO rank

Classement TACT effectué par la même personne - TACT rank made by the same tester. Symboles représentant les éprouvettes sur les figures 4 a et 4 b - Symbols for samples in $4 \mathrm{a}$ and $4 \mathrm{~b}$ figures.

Ponçage grossier ou sans ponçage - Rough sanding or without sanding.

Ponçage fin - Smooth sanding.

son toucher rugueux eu égard à sa beauté (peut-être plutôt à son aspect régulier), alors qu'il serait plus sévère avec le chêne rouge, grossier et hétérogène.

Quoi qu'il en soit, la dérive systématique que nous observons entre les deux modalités nous incite à penser que la lumière joue effectivement un rôle, en partie masqué dans le cas présent, mais qui pourrait devenir moins négligeable en d'autres circonstances. Il convient donc d'être prudent lorsque l'on désire exploiter ou compléter des résultats obtenus dans des conditions expérimentales inconnues.

\section{Conclusion}

La comparaison de la méthode pneumatique et des deux méthodes sensoriclles d'estimation de la rugosité nous a permis de confirmer ou de mettre en évidence plusieurs qualités du rugosimètre pneumatique : 
- rapidité : une journée a suffi pour effectuer les 18 répétitions (au total plus de 500 mesures). Les modalités visiotactiles ont demandé quant à elles plusieurs jours de préparation, puis une dizaine de jours environ pour les tests proprement dits en raison du manque de disponibilité des personnes sollicitées. Un test pneumatique (36 mesures) demande environ 3 mesures de travail effectif, soit 2 à 5 fois moins qu'un test sensoriel.

- facilité d'emploi : aucune compétence particulière n'est requise pour manipuler le pneumomètre et nous n'avons observé aucune influence de l'opérateur sur les résultats. Les tests visiotactiles que nous avons expérimentés ne demandent pas davantage de compétences, mais exigent une plus forte concentration. Plusieurs personnes doivent être mises à contribution, ne serait-ce qu'en raison de la lassitude que provoqueraient des tests répélés.

- fiabilité : la répétabilité du classement pneumatique est supéricure a celle d'un classement manuel. Nous avons cependant constaté l'existence d'un seuil de rugosité en deçà duquel le pouvoir de ségrégation de l'appareil pneumatique décroît considérablement.

Ces qualités font du rugosimètre un outil intéressant, mais un problème majeur subsiste puisque les mesures effectuées montrent que la rugosité telle que la perçoit la peau humaine diffère sensiblement de ce que nous avons appelé la "rugosité pneumatique». L'analyse des données nous laisse supposer que les défauts de surfaces n'interviennent pas de la même façon dans l'un et l'autre cas; la présence de gros vaisseatix striant la surface se traduirait par une forte "rugosité pneumatique ", alors que les arrachements de surface résultant d'un ponçage défectueux seraicnt surtout perceptibles alu toucinu (tabl. 4).

TIBLLAU 4

Comparaison des rugosités sensorielle et pneumatique pour deux profils théoriques.

Comparison of sensorial and monmaticat roughess for wo theoretical profiles.

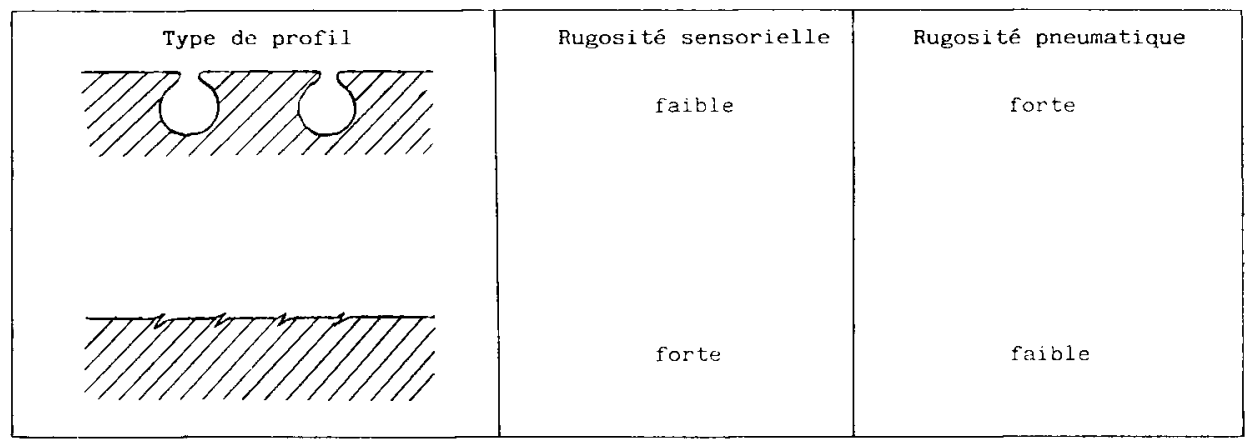

Rugosité sensoriclle - Sensorial ronghness.

Rugosité pneumatique - Pneumatic roughness.

Faible - Simall.

Forte - High. 
Il serait nécessaire, pour vérifier cette hypothèse, de réaliser une étude complémentaire où seraient confrontées aux mesures pneumatiques les données fournies par un rugosimètre à palpeur capable de discerner la part de rugosité imputable au réseau vasculaire et celle provoquée par l'usinage.

L'évaluation de la qualité d'une finition est l'une des applications les plus courantes pour un rugosimètre. Si nos conclusions reflètent effectivement la réalité, un tel débouché ne serait pas concevable pour le pneumomètre. Son exploitation en industrie serait limitée aux emplois pour lesquels la structure du bois doit être prise en considération, par exemple dans le cas du collage. C'est surtout en laboratoire qu'un tel appareil pourrait devenir un outil efficace, en particulier lorsque l'on s'attache à évaluer l'effet des propriétés du bois sur la rugosité, auquel cas la variabilité provoquée par l'usinage doit être éliminée. C'était en particulier le cas de Marchal (1983, op. cit.) qui, pour étudier l'effet de la vitesse de croissance et de la densité du bois sur la rugosité des placages tranchés de chêne, a utilisé un rugosimètre pneumatique de conception proche de celui que nous avons expérimenté ici.

Regu en juin 1984.

Accepté en mars 1985.

\begin{abstract}
Summary
Comparative study of three methods for grading solid wood according to roughness using pneumatic or sensoriel procedures
\end{abstract}

18 samples of solid wood representing three species and five surface qualities were graded according to roughness (radial and longitudinal face) by using three procedures :

VISO : classical visual and tactile test;

TACT : tactile test in darkness;

PNEU : pneumatic roughnessmeter (by measuring of air flow).

Results analyzed by correspondence analysis showed the excellent replicability of data recorded by the pneumatic device. However the latter system was more sensitive to the wood structure at the surface (particularly the occurence of large vesscls) than to the milling characteristics. Besides, the effect of light on visual and tactile grading was demonstrated. The samples of bird cherry appeared to be rougher in the dark whereas the roughness of red oak was overestimated in the light.

\title{
Zusammenfassung
}

Versuch und vergleich dreier Einstufungsmethoden der oberflachen von Massivholz im Hinblick auf ihre Rauheit Pneumatische und Tastmethoden

Achtzehn Massivholzproben, die drei Baumarten gekreuzt mit fünf Verarbeitungsmethoden darstellten, wurden mittels drei Methoden nach ihrer Rauheit eingestuft (Querschnitt und Längsschnitt).

VISO : üblicher Sch-und Tasttest ;

TACT : reiner Tasttest, im Dunkeln durchgeführt ;

PNEU : pneumatischer Rauheitsmesser (durch Luftausströmen). 
Die Ergebnisse, dic mit der Faktorenanalyse verarbeitet wurden, zcigen im Hinblick auf die Methodik, dass die Wiederholbarkeit der vom pneumatischen Apparat erbrachten Messungen ausgezeichnet ist, aber dass letzterer sich gegenüber den Eigenschaften der Holzoberfläche (insbesondere Bestehen von dicken Gefässen) empfindlicher zeigt, als gegeniiber den Eigenschaften der Verarbeitung. Andererseits konnte ein Einfluss des Lichtes auf die Seh-Tasteinstufung nachgewiesen werden : die Proben von Prunus avium erscheinen rauher ohne Licht, wogegen die Rauheit von Quercus rubra überbewertet wird, wenn die Proben sichtbar sind.

\section{Références bibliographiques}

Bonac T., 1975. Measuring of wood surface texture by the pneumatic method. Paperi ja pult, $n^{\prime \prime} 4,309-326$.

BONAC T., 1979. Wood roughness volume and depth estimated from pneumatic surface measurements. Wood Science, 11 (4), 227-232.

Marchal R., 1983. Intérêt de la prise en compte de caractéristiques physiques et anatomiques simples du bois de chêne pour l'appréciation de la qualité des placages d'ébénisterie. D.E.A. «Sciences du Bois», 1.N.P.L., Université de Nancy 1, Station de Recherches sur la Qualité des Bois, C.N.R.F., Nancy-Champenoux, octobre.

Sadoh T., Takeucil M., Nakato K., 1977. Relatior.ships between sensory and physical cvaluations of wood surface roughness. Bulletin of the Kyoto University Forests, $\mathrm{n}^{\circ} 49$, October, 138-144.

Schmaltz G., 1936. Technische Oberflachenkunde. Springer Verlag, Berlin.

Yasuda A., Sadoh T., Nakato K., 1983. Visual and tactile roughness of hardwood surfaces relating to physical roughness. Mokuzai Gakkaishi, vol. 29, n"11, 731-737. 